\title{
Low-loss high-fidelity frequency beam splitter with tunable split ratio based on electromagnetically induced transparency
}

\author{
Kao-Fang Chang, ${ }^{1}$ Ta-Pang Wang, ${ }^{1}$ Chun-Yi Chen, ${ }^{1}$ Yi-Hsin Chen,,${ }^{2,5}$ Yu-Sheng Wang, ${ }^{1}$ Yong-Fan Chen, $, 3,5$, \\ Ying-Cheng Chen, ${ }^{4,5}$ and Ite A. Yu (1) ${ }^{1,5}{ }^{*}$ \\ ${ }^{1}$ Department of Physics, National Tsing Hua University, Hsinchu 30013, Taiwan \\ ${ }^{2}$ Department of Physics, National Sun Yat-sen University, Kaohsiung 80424, Taiwan \\ ${ }^{3}$ Department of Physics, National Cheng Kung University, Tainan 70101, Taiwan \\ ${ }^{4}$ Institute of Atomic and Molecular Sciences, Academia Sinica, Taipei 10617, Taiwan \\ ${ }^{5}$ Center for Quantum Technology, Hsinchu 30013, Taiwan
}

(Received 27 June 2019; accepted 5 January 2021; published 29 January 2021)

\begin{abstract}
A frequency beam splitter (FBS) with the split ratio of 0.5, i.e., 50/50 FBS, can be used as the frequency-mode Hadamard gate for frequency-encoded photonic qubits. A FBS with the split ratio of 1 is exactly the coherent frequency converter (CFC) for frequency up or down conversion of photons. Previous works revealed that all kinds of 50/50 FBS and CFC operating at the single-photon level had overall efficiency or output-to-input ratio around $50 \%$ or less. In this work, our 50/50 FBS and CFC are made with the four-wave mixing (FWM) process based on the double- $\Lambda$ electromagnetically induced transparency (EIT) scheme. We achieved an overall efficiency of $90 \pm 4 \%$ in the 50/50 FBS and that of $84 \pm 4 \%$ in the CFC using coherent-state single photons, both of which are the best up-to-date records. Furthermore, we utilize the scheme of Hong-Ou-Mandel interference (HOMI) to measure the fidelity or degree of coherence of the FBS. The fidelity indicated by the HOMI's $g^{(2)}$ measurement of the $50 / 50$ FBS is $0.99 \pm 0.01$. This high fidelity demonstrates the low noise of the frequency conversion in the EIT-based FWM process. Such low-loss high-fidelity FBS with the tunable split ratio can lead to useful operations or devices in long-distance quantum communication.
\end{abstract}

DOI: 10.1103/PhysRevResearch.3.013096

\section{INTRODUCTION}

Quantum information or wave functions is commonly encoded in photons' polarization or spatial mode. Compared with these two kinds of photonic qubits, frequency-encoded qubits [1-6], in addition to time-bin-encoded qubits [7-11] and dual-rail-encoded qubits [12-14], are more stable over long transmission distances and more robust against birefringent materials. Among quantum logic operations, the Hadamard gate is an essential component. A beam splitter is exactly the Hadamard gate for spatial-mode qubits. In the context of frequency-encoded photonic qubits, a frequency beam splitter (FBS) is the Hadamard gate. In this work, we demonstrate a low-loss high-fidelity FBS with a tunable split ratio, where the split ratio is the ratio of photon number in one output frequency mode to total output photon number. At the split ratio of 0.5 , a FBS (named 50/50 FBS) can be employed as the frequency-mode Hadamard gate. At the split ratio of 1, a FBS (named coherence frequency converter or CFC) can be operated to coherently convert photonic qubits from one frequency or wavelength to another in quantum commuincation.

\footnotetext{
*yu@phys.nthu.edu.tw; yfchen@mail.ncku.edu.tw

Published by the American Physical Society under the terms of the Creative Commons Attribution 4.0 International license. Further distribution of this work must maintain attribution to the author(s) and the published article's title, journal citation, and DOI.
}

To date, all kinds of the 50/50 FBS and CFC operating at the single-photon level had output-to-input ratios or overall efficiencies (including decay due to propagation or insertion loss in media, input coupling efficiency, frequency conversion efficiency, etc.) around $50 \%$ or less [5,6,15-25]. Most of these works suffered large insertion loss induced by media, which not only reduces the output-to-input ratio but also may lead to additional quantum noise. Here, our low-loss FBS is made with the four-wave mixing (FWM) process based on the double- $\Lambda$ electromagnetically induced transparency (EIT) scheme [26-33]. Using the transition scheme depicted in Fig. 1(a), we converted a coherent-state single photon in the 780-nm mode to another photon in the superposition of 780- and 795-nm modes, and demonstrated that the 50/50 FBS has an output-to-input ratio of $90 \pm 4 \%$. Furthermore, we performed the CFC from 780 to $795 \mathrm{~nm}$ with light pulses of photon number less than 1 , and achieved an output-to-input ratio of $84 \pm 4 \%$. Both output-to-input ratios are the best upto-date records.

To test the fidelity of a quantum device or process, one should perform quantum process tomography [34-37]. Outcome of quantum process tomography depends on the degree of coherence of the device or process. Here we utilized the Hong-Ou-Mandel interference (HOMI) to measure the degree of coherence of the FBS [38-42]. The HOMI is a two-photon phenomenon, in which one two-mode wave function formed by the two outputs of the FBS interferes with another. In the HOMI measurement of our 50/50 FBS, the value of normalized cross correlation function, $g^{(2)}$, reveals that the fidelity 

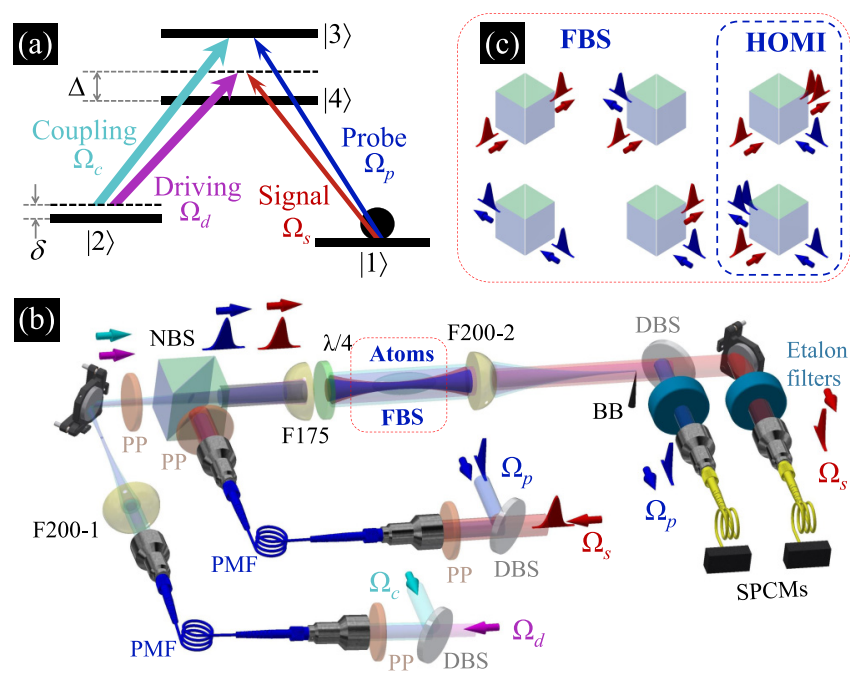

FIG. 1. Transition diagram, experimental setup, and operation of FBS. (a) Relevant energy levels and laser excitations in the experiment. $|1\rangle,|2\rangle,|3\rangle$, and $|4\rangle$ represent the states $\mid 5 S_{1 / 2}, F=1, m=$ $1\rangle,\left|5 S_{1 / 2}, F=2, m=1\right\rangle,\left|5 P_{3 / 2}, F=2, m=2\right\rangle$, and $\mid 5 P_{1 / 2}, F=$ $2, m=2\rangle$ of ${ }^{87} \mathrm{Rb}$ atoms, respectively. The coupling $\left(\Omega_{c}\right)$ and probe $\left(\Omega_{p}\right)$ fields drove the transitions nearly resonantly, and the driving $\left(\Omega_{d}\right)$ and signal $\left(\Omega_{s}\right)$ fields drove the transitions with a detuning of $\Delta$. A small two-photon detuning in some measurmenets is donoted as $\delta$ in the figure. (b) Schema of the experimental setup. DBS: dichroic beam splitter; PP: polarizer or polarizing beam splitter with half-wave plate; PMF: polarization-maintained optical fiber; PBS: polarizing beam splitter; NBS: nonpolarizing beam splitter with $T / R$ $=90 / 10$; F200-1, F175, F200-2: lenses with focal lengths of 200, 175 , and $200 \mathrm{~mm} ; \lambda / 4$ : quarter-wave plate; BB: beam block; SPCM: single-photon counting module. (c) Illustration of 50/50 FBS. The left four diagrams depict that a 780-nm (or 795-nm) photon arriving to the input can result in either a 780-nm photon or a 795-nm photon with the equal probability at the output. The right two diagrams depict that a 780-nm and a 795-nm photons simultaneously arriving to the input can produce two photons of the same wavelength at the output due to the Hong-Ou-Mandel interference.

is $0.99 \pm 0.01$. Since two phase-uncorrelated single photons of the coherent state were utilized in the measurement, the result is interpreted as the classical fidelity of the FBS. The high fidelity or degree of coherence of the 50/50 FBS also suggests that a quantum state can be well preserved in the double- $\Lambda$ EIT scheme. The EIT mechanism is universal and can work for various media [43-47]. Hence, the high-fidelity low-loss FBS reported here can be readily applied to systems of the optical depth and decoherence rate similar to those in this work.

\section{EXPERIMENTAL SETUP}

Our experiment was carried out with laser-cooled ${ }^{87} \mathrm{Rb}$ atoms [48-50]. Figure 1(b) shows the schema of experimental setup. In the photon-atom coupling scheme as depicted in Fig. 1(a), the 780-nm probe and coupling fields form the first EIT configuration under the one-photon resonance; the 795-nm signal and driving fields form the second one with a large one-photon detuning, $\Delta$. The coupling and driving fields were strong quasi-continuous-wave light. The probe and signal fields were weak classical pulses, or coherent-state single- or few-photon pulses. Other details of the experimental system can be found in Appendix A.

To characterize our experimental system and verify measurement outcomes, we made theoretical predictions with the optical Bloch equations (OBEs) of atomic density matrix and the Maxwell-Schrödinger equations (MSEs) of light fields, which can be found in Appendix B. In these equations and thorough the paper, $\Omega_{c}, \Omega_{d}, \Omega_{p}$, and $\Omega_{s}$ denote the Rabi frequencies of the coupling, driving, probe, and signal fields, $\delta$ is the two-photon detuning of the Raman transition between two ground states $|1\rangle$ and $|2\rangle, \gamma$ represents the ground-state decoherence rate, $\Gamma$ denotes the spontaneous decay rate of the excited states $|3\rangle$ and $|4\rangle$ which is about $2 \pi \times 6 \mathrm{MHz}$ in our case, and $\alpha$ is the optical depth (OD) of the medium. The measurements that determined $\Omega_{c}, \Omega_{d}, \gamma$, and $\alpha$ in the experiment are illustrated in Appendix C.

The split ratio here is defined as the ratio of 795-nm output photon number to total output photon number under the condition that only the $780-\mathrm{nm}$ photons are present at the input. Tuning the split ratio of FBS can be done by varying either two-photon detuning $\delta$ or one-photon detuning $\Delta$. In this study of split ratio, only the $780-n m$ probe pulse of classical light was present at the input, and $\Omega_{c}=\Omega_{d}$. A part of the $780-\mathrm{nm}$ input pulse was converted to the 795-nm signal pulse at the output, and the remaining became the 780-nm output pulse. Figures 2(a) and 2(b) show the energy transmissions of 780- and 795-nm output pulses as functions of the two-photon detuning $\delta$ and the one-photon detuning $\Delta$, respectively. One can see that using $\delta$ to tune the split ratio can suffer a larger loss, and using $\Delta$ is more efficient. In Fig. 2(b), the split ratio can be tuned from 1 to 0.5 or smaller with $|\Delta| /(2 \pi) \geqslant$ $130 \mathrm{MHz}$. The total energy transmission of 780- and 795-nm output pulses is $85 \%$ (or $88 \%$ ) at the split ratio equal to 0.97 (or 0.54). A smaller split ratio results in a higher total transmission. In both figures, the apparent phenomenon of oscillation indicates that the underlying mechanism of FWM involves the interference effect [30,31]. The theoretical predictions were calculated by numerically solving OBEs and MSEs with the experimentally determined parameters of $\alpha$, $\Omega_{c}, \Omega_{d}$, and $\gamma[49,50]$. Consistency between the experimental data and theoretical predictions is satisfactory.

To test whether the scheme of our FBS can also work well at the single-photon level, we performed the measurements with coherent-state pulses of photon number equal to or less than 1. Two etalon filters were installed to provide the extinction ratio of $43 \mathrm{~dB}$. The etalons, together with the scheme of spatial filter (see the third paragraph in Appendix A), can effectively block the strong coupling and driving light from entering single-photon counting modules (SPCMs). Two Excelitas SPCM-AQRH-13-FC were used to detect the 780- and 795-nm output photons. The collection efficiencies (including the SPCM's quantum efficiency) of the 780- and 795-nm photons were about 0.13 and 0.12 for the data in Fig. 3(a) [0.17 and 0.12 for those in Fig. 3(b)]. We had another SPCM at the input to monitor the input photon number. All of the photo multiplier tubes used in the measurements of classical-light pulses and the SPCMs used in those of singlephoton or few-photon pulses were calibrated to account for 

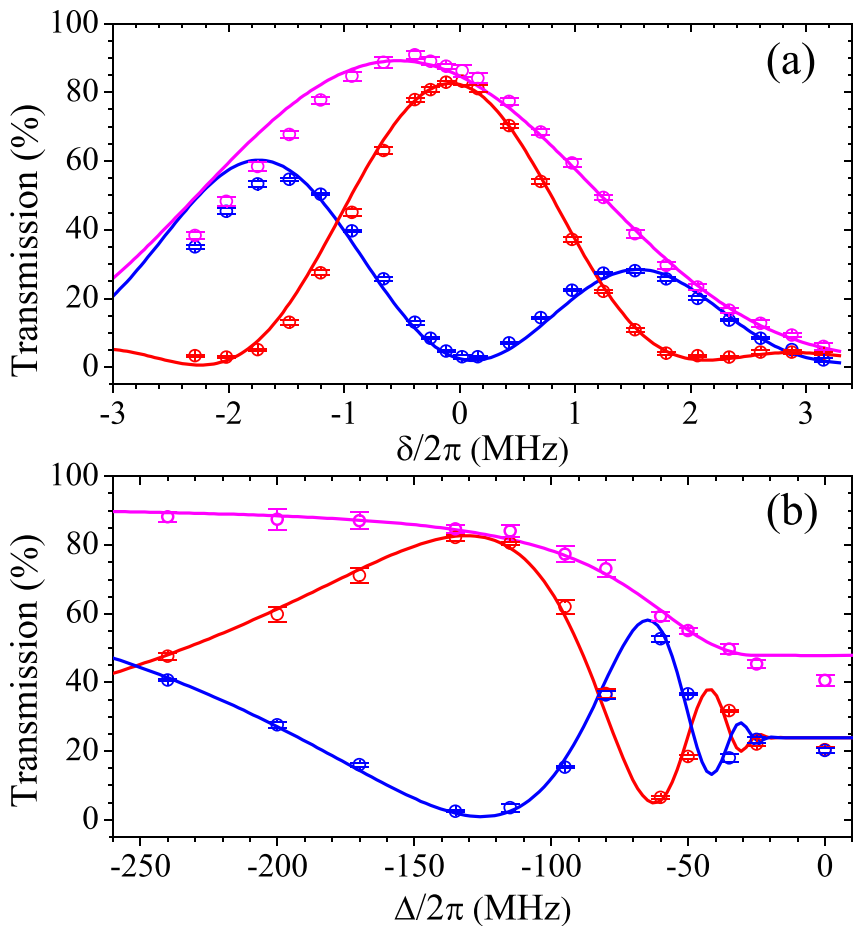

FIG. 2. Tuning the split ratio of frequency beam splitter. (a) At one-photon detuning $\Delta=-2 \pi \times 135 \mathrm{MHz}$, transmissions as functions of two-photon detuning $\delta$, where $\delta=0$ is defined by the maximum transmission of the output signal pulse, but not by making the two-photon Raman transition of $|1\rangle \rightarrow|2\rangle$ resonant. (b) At $\delta=0$, transmissions as functions of $\Delta /(2 \pi)$. In (a) and (b), only the 780 -nm probe pulse with the $e^{-2}$ full width of $3.0 \mu$ s was present at the input. Blue, red, and magenta circles are the experimental data of 780-nm probe and 795-nm signal output transmissions, and their total transmission, respectively. Solid lines are the theoretical predictions calculated with $\Omega_{c}=\Omega_{d}=3.0 \Gamma, \alpha(\mathrm{OD})=130$, and $\gamma=3 \times 10^{-3} \Gamma$, which were experimentally determined by the method illustrated in Appendix C. The asymmetry between positive and negative values of $\delta$ is due to the existence of a phase mismatch in the experimental system.

different detection efficiencies between the wavelengths or between the detectors. In each SPCM's counting, it took $0.15 \mathrm{~s}$ to replenish cold atoms, switch off the magneto-optical trap (MOT), perform the temporal dark-MOT, and optically pump all population to a single Zeeman state, before the input pulse was fired.

\section{RESULTS AND DISCUSSION}

At the split ratio of 1 , the FBS acts like a CFC transforming 780-nm photons completely into 795-nm photons. Figure 3(a) shows SCPM counts of input and output photons as functions of time. The best fit of the data in Fig. 3(a) is consistent with the result of classical light shown by Fig. 5(c) in Appendix C. The baseline count was mainly contributed from the leakage of strong coupling or driving fields. Using the area below the best fit but excluding the baseline count, we determined that the overall conversion efficiency from the 780-nm single photons to the 795-nm single photons or the output-to-input
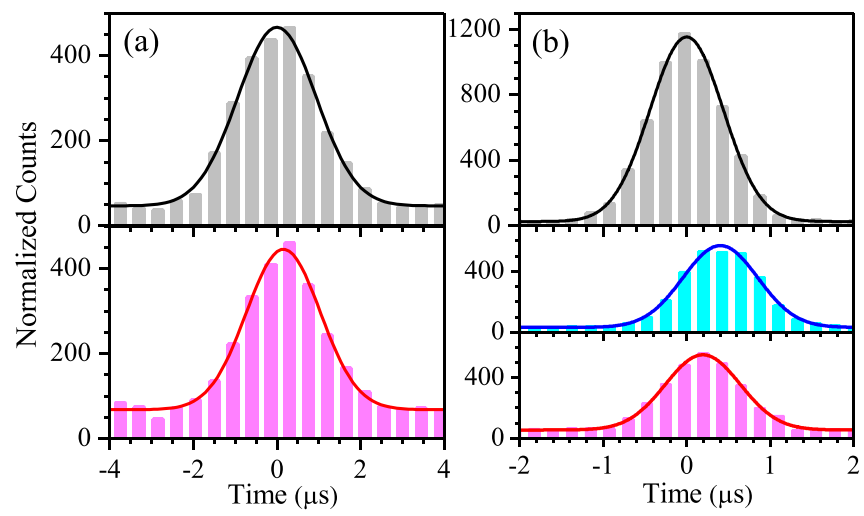

FIG. 3. Operations of CFC in (a) and 50/50 FBS in (b) using coherent-state single-photon-level pulses. In (a), the number of input photon per pulse was 0.68 , and the split ratio $\sim 1$ set by $\Delta /(2 \pi)=$ $-125 \mathrm{MHz}$. Top: counts of 780-nm input photons; bottom: those of 795-nm output photons. In (b), the number of input photon per pulse was 1.0 , and the split ratio $\sim 0.5$ set by $\Delta /(2 \pi)=-210 \mathrm{MHz}$. Top: counts of 780-nm input photons; middle, and bottom: those of 780and 795-nm output photons. In (a) or (b), the width of time bin for SPCM counts was $450 \mathrm{~ns}$ or $225 \mathrm{~ns}$, and the data were the results of 24000 or 32000 measurements. All of black, red, and blue lines are the Gaussian best fits. Excluding the baseline count, the ratios of output to input photon numbers are $84 \pm 4 \%$ in (a) and $90 \pm 4 \%$ in (b).

ratio is $84 \pm 4 \%$. The input and output reference points are right before and after the atom cloud, respectively.

The split ratio of 0.5 can make a $50 / 50$ FBS or potentially a Hadamard gate for frequency-encoded photonic quits. In Fig. 3(b), SCPM counts of 780-nm input photons and those of 780-nm and 795-nm output photons are plotted against time. The best fits of the data in Fig. 3(b) are consistent with the results of classical light shown by Fig. 6(a) in Appendix D. The comparison between Figs. 3(a) and 3(b) indicates that employing single photons of a narrower temporal width can increase the amplitude-to-baseline ratio, while the output-toinput ratio was nearly intact. In our 50/50 FBS, the total transmission or ratio of total output photons to input photons is $90 \pm 4 \%$.

We have now made the FBS which can operate with single photons. In analogy to an ordinary beam splitter (BS), 780-nm (or 795-nm) input photons are reflected into 795-nm (or 780nm) output photons and transmitted into 780-nm (795-nm) output photons by our FWM-based FBS, with the split ratio defined by the ratio of reflected output photon number to total output photon number. Figure 1(c) illustrates the operation of 50/50 FBS. The next question is whether this FBS can potentially be suitable for quantum information processing. To answer the question, fidelity $F$ is the important issue and can be determined by the following formula $[6,51,52]$ :

$$
F=\frac{\left|\operatorname{Tr}\left[\hat{V}^{\dagger} \hat{U}\right]\right|^{2}}{4 T},
$$

where $\hat{U}$ represents the operator of an ideal BS, $\hat{V}$ represents the operator of FWM-based FBS in the case here, $\operatorname{Tr}[\ldots]$ means the operation of trace, and $T$ is the total transmission or success probability of $\hat{V}$. 
Considering the FBS, we define $t_{1}\left(t_{2}\right)$ and $r_{1}\left(r_{2}\right)$ as the transmission and reflection coefficients of input 1 (input 2), and $\phi_{1}\left(\phi_{2}\right)$ as the phase difference between the reflected and transmitted outputs. The general expression of $\hat{V}$ is given by

$$
\left[\begin{array}{cc}
t_{1} & r_{2} e^{i \phi_{2}} \\
r_{1} e^{i \phi_{1}} & t_{2}
\end{array}\right]
$$

The split ratio of two inputs are determined by $r_{1}^{2} /\left(t_{1}^{2}+r_{1}^{2}\right)$ and $r_{2}^{2} /\left(t_{2}^{2}+r_{2}^{2}\right)$. In reality, the ground-state decoherence rate $\gamma$ in the experimental system was not negligible, making two inputs produce different split ratios and $\phi_{1} \neq \phi_{2}$. An ideal $50 / 50$ BS must have $\phi_{1}+\phi_{2}=\pi$. Corresponding to $\hat{V}$ in Eq. (F2) of a realistic BS with two split ratios close to 0.5, $\hat{U}$ of the ideal $50 / 50 \mathrm{BS}$ can be written as $[53,54]$

$$
\hat{U}=\frac{1}{\sqrt{2}}\left[\begin{array}{cc}
1 & e^{i(\pi-\Delta \phi) / 2} \\
e^{i(\pi+\Delta \phi) / 2} & 1
\end{array}\right],
$$

where $\Delta \phi \equiv \phi_{1}-\phi_{2}$. The derivation in Appendix E shows that the fidelity of $\hat{V}$ is

$$
F=\frac{1}{2}+\frac{\bar{t} \bar{r}}{T} \sin \left(\frac{\phi}{2}\right),
$$

where $\bar{t}=\left(t_{1}+t_{2}\right) / 2, \bar{r}=\left(r_{1}+r_{2}\right) / 2, T=\bar{t}^{2}+\bar{r}^{2}$, and $\phi=$ $\phi_{1}+\phi_{2}$.

According to Eq. (F10), one can immediately see that the phase $\phi$ approaching to $\pi$ can make a high-fidelity FBS. To determine $\phi$, we employed the Hong-Ou-Mandel interference (HOMI) [38-42], and measured the normalized cross correlation function, $g^{(2)}$, between two outputs of the FBS. A simple example, to explain why the HOMI measurement can determine $\phi$ of Eq. (F10), is illustrated in Appendix F. In the HOMI, it is well known that, with a 50/50 BS in the ideal condition, two Fock-state single photons results in $g^{(2)}=0$ [38,39], and two phase-uncorrelated coherent-state single photons results in $g^{(2)}=0.5$ [40-42]. Here we sent two pulses to the two input ports of 50/50 FBS in the HOMI measurement. Each pulse consisted of a coherent-state single photon or few photons. The wavelength of one pulse was $780 \mathrm{~nm}$ and that of the other was $795 \mathrm{~nm}$. Since the two pulses had the same mean photon number and were phase uncorrelated, the derivation in Appendix G shows that $g^{(2)}$ of the two output ports is given by

$$
g^{(2)}=1+\frac{2 t_{1} t_{2} r_{1} r_{2}}{\left(t_{1}^{2}+r_{2}^{2}\right)\left(t_{2}^{2}+r_{1}^{2}\right)} \cos \phi .
$$

In Fig. $4, g^{(2)}$ is plotted against the delay time between the two input pulses. The minimum $g^{(2)}$ is $0.53 \pm 0.03$ and occurs at the delay time of $200 \mathrm{~ns}$. Based on the data shown in Figs. 6(a) and 6(b) of Appendix D, this 200-ns delay time is expected. The two-photon event of both photons from two input ports transmitting through the FBS, and that of both photons being reflected by the FBS were nearly indistinguishable under such delay time. Right before taking the data in Fig. 4, we measured the data similar to those in Figs. 6(a) and 6(b), and found $t_{1}^{2}=46 \%, r_{1}^{2}=46 \%, t_{2}^{2}=51 \%$, and $r_{2}^{2}=39 \%$. The minimum $g^{(2)}$ and Eq. (5) result in $\cos \phi=-0.94(6)$. Finally, we use Eq. (F10) and the above values of $t_{1}, r_{1}, t_{2}, r_{2}$, and $\phi$ to determine $F=0.99 \pm 0.01$, indicating that the FWM-based FBS possesses excellent fidelity.

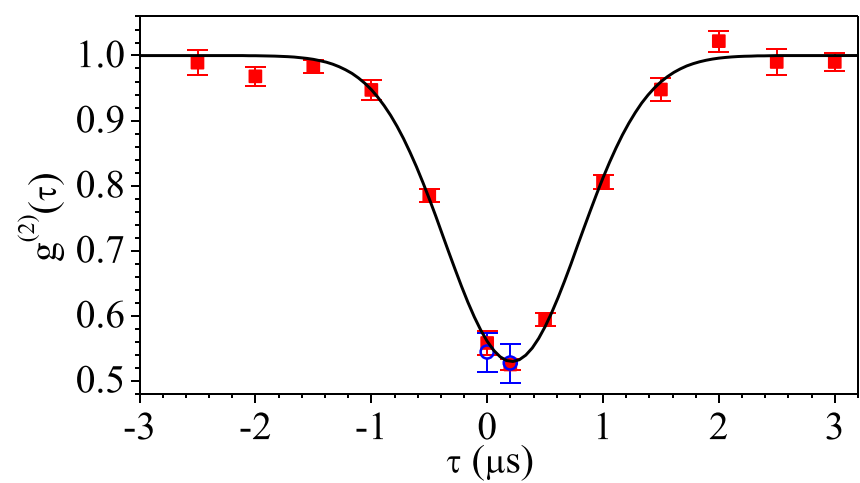

FIG. 4. Measurement of cross correlation function $g^{(2)}$ in the Hong-Ou-Mandel interference. $g^{(2)}$ is plotted against the delay time between two input pulses, $\tau$. The experimental condition was very similar to that in Fig. 6 of Appendix D. Blue circles are the experimental data taken at $\Delta /(2 \pi)=-215 \mathrm{MHz}$ with two single-photon input pulses of 780 and $795 \mathrm{~nm}$. Each circular data point, in which the contribution from background counts is removed, is the result of 28800 measurements. Red squares are the experimental data taken at $\Delta /(2 \pi)=-205 \mathrm{MHz}$ with two five-photon input pulses. Each square data point, corrected for the saturation effect of SCPM's gain and the background contribution, is the result of 9600 measurements. The two values of $\Delta$, which set the split ratio of FBS to $\sim 0.5$, are different due to the day-to-day variation of OD. The $e^{-2}$ full width of input pulses was $1.7 \mu$ s. The time windows for SPCM counts of the blue circular and red square data points were 1.8 and $8.0 \mu \mathrm{s}$, respectively. Solid line is the best fit of a Gaussian function with the $e^{-2}$ full width of $2.3 \mu$ s and the minimum $g^{(2)}$ of 0.53

We compared the similarity between the ideal and actual processes of 50/50 FBS. The degree of similarity consists of two decisive factors. One factor is the comparison between the ideal and actual split ratios, and the other is the determination of degree of coherence of the actual FBS process. The fidelity defined by Eq. (F1) or (F10) clearly exhibits these two factors. As shown by Eq. (F10), the degree of coherence is indicated by the phase $\phi\left(=\phi_{1}+\phi_{2}\right)$ of the FBS, where $\phi_{1}$ or $\phi_{2}$ is the phase difference between the frequency-converted and frequency-unchanged photons. In the language of a standard spatial-mode beam splitter, $\phi_{1}$ or $\phi_{2}$ is the phase difference between the reflected and transmitted photons. This prompts us to employ the HOMI to determine this phase $\phi$, i.e., the degree of coherence of the FBS. Since the coherent-state single photons were utilized in the measurement, the result here is interpreted as the classical fidelity of the FBS.

There can be noise caused by the Raman process $[55,56]$, the spontaneous emissions of excited states, etc., within the FBS operation. Such noise will be added to the output probe and signal beams, making $g^{(2)}$ of the coherent-state HOMI measurement greater than the ideal value of 0.5 . We were not able to identify this type of noise directly from the data. Nevertheless, since $g^{(2)}=0.53$ in the HOMI measurement or the classical fidelity is 0.99 , we believe that the noise generated within the FBS process is a little. Such high fidelity also demonstrates the frequency conversion of EIT-based FWM process exhibits low noise. 


\section{CONCLUSION}

In conclusion, utilizing the EIT-based FWM process we experimentally demonstrated a FBS of the tunable split ratio with coherent-state single-photon pulses. At the split ratio $\sim 1$, the FBS converted all of 780-nm input photons to 795-nm output photons with the output-to-input ratio of $84 \pm 4 \%$. Both of the output-to-input ratios or overall efficiencies are the best up-to-date records. Discussion on the FBS bandwidth can be found in Appendix E [44,57-60]. To test the fidelity of the FBS, we utilized the HOMI to determine the degree of coherence. The value of $g^{(2)}$ in the HOMI measurement indicates that the classical fidelity of our 50/50 FBS is $0.99 \pm 0.01$, which suggests a quantum state can be well preserved in the EIT scheme. This low-loss high-fidelity FBS can lead to useful devices or operations, such as entanglement swapping and multiplexing, in long-distance quantum communication.

\section{ACKNOWLEDGMENTS}

This work was supported by the Ministry of Science and Technology of Taiwan under Grants No. 106-2119-M-007003, No. 107-2745-M-007-001, No. 108-2639-M-007-001ASP, and No. 109-2639-M-007-002-ASP.

\section{APPENDIX A: EXPERIMENTAL SYSTEM}

The cold ${ }^{87} \mathrm{Rb}$ atoms in the experiment were produced by a MOT. There were about $3 \times 10^{9}$ in the cigar-shaped atom cloud of the dimension of $14 \times 2 \times 2 \mathrm{~mm}^{-3}$ [48]. Before each measurement, we optically pumped all population to the Zeeman states of $|F=1, m=1\rangle$ and $|F=2, m=2\rangle$ [3]. The population in $|F=2, m=2\rangle$ is irrelevant to this study, and all laser fields in the FWM measurement did not drive this state. Figure 1(a) shows the transitions driven by the probe, coupling, driving, and signal fields, all of which had the $\sigma+$ polarization, and propagated along the major axis of the atom cloud. The OD of the probe and signal transitions was about $\sim 100-130$. The wavelength of the probe and coupling fields is $780 \mathrm{~nm}$, and that of the driving and signal fields is $795 \mathrm{~nm}$. We used light from a master laser to seed the probe and coupling lasers for the stabilization of frequency difference between the two lasers [50]. An electro-optic modulator (EOM) was installed in one of the seeding paths. The first-order sideband of the EOM was employed for the seeding, which produces a frequency difference of about $6.8 \mathrm{GHz}$ between the probe and coupling lasers. We made the similar arrangement to seed the signal and driving lasers. Therefore, the decoherence rate caused by fluctuation of the frequency difference between the probe and coupling fields (or between the signal and driving fields) was little.

The coupling and driving beams came out of a polarization-maintained (PM) optical fiber before entering the atom cloud, and the probe and signal beams came out of another PM optical fiber. This can ensure the coupling and driving beams (the probe and signal beams) to spatially overlap well. The $e^{-2}$ full width of the coupling and driving beams was $2.0 \mathrm{~mm}$, and that of the probe and signal beams was $0.2 \mathrm{~mm}$. We arranged an angle separation of about $0.9^{\circ}$ between the coupling (driving) and the probe (signal) prop- agation directions. This angle separation is small enough to make negligible decoherence rate [61,62], and is also large enough to prevent the coupling and driving light from entering the probe's and signal's SPCMs.

A scheme of spatial filter was also used to block the coupling and driving light. In Fig. 1(b), the coupling and driving beams first passed through F200-1 before merged with the probe and signal beams at NBS, then became quasiplane waves after F175, and finally were focused by F200-2 and blocked by BB at the focal point. The probe and signal beams were focused to the center of the atom cloud by F175, and transformed to quasiplane waves by F200-2. Because of the angle separation, BB which is actually an iris diaphragm caused little attenuation of the probe and signal light.

The input and output reference points of the FBS are right before and after the atom cloud, respectively. The collection efficiency of the probe or signal detection system was $\sim 12$ $18 \%$. Referred to Fig. 1(b) in the main text, this efficiency is resulted from the transmittance of the dichroic beam splitter (DBS before the two etalon filters) of $95 \pm 1 \%$, the coupling efficiencies of single-mode optical fibers before (not shown in the figure) and after the etalon filters of $60 \pm 5 \%$ and $72 \pm 6 \%$, respectively, the etalon transmittance of $65 \pm 5 \%$, and the SPCM's quantum efficiency of about $50 \%$ (or $55 \%$ ) for 780- (or 795-) nm light. Without the atoms, the transmittance from the lenses F175 to F200-2 is $94 \pm 0.6 \%$. The two polarization-maintained fibers (PMF) in Fig. 1(b) were used to keep the stability of optical paths of light fields, and the coupling efficiency into the PMF was $\sim 50-60 \%$. We used a DBS before each PMF to couple the two light fields of different frequencies into the same spatial mode. The attenuation caused by the DBS is $5 \pm 1 \%$. The above independently measurable losses are not intrinsic in the FBS operation and, thus, are not included in the output-to-input ratio or overall conversion efficiency of the FBS.

Several technical issues involved in the low-loss FWM process. First, a medium's OD $(\alpha)$ must be sufficiently large. The conversion efficiency from one wavelength to another can be enhanced by OD, shown by Eq. (C1) in Appendix C. A larger OD can also make the 50/50 FBS have a higher total transmission and a better fidelity as illustrated in Sec. 2 of Appendix F. Second, the wavelength conversion process requires the phase match, i.e., $\Delta k \equiv\left(\vec{k}_{p}-\vec{k}_{c}+\vec{k}_{d}-\vec{k}_{s}\right) \cdot \hat{z}=$ 0 , where $\vec{k}_{x}$ represents the wave vector of light field. A phase mismatch, i.e., $\Delta k \neq 0$, can cause the loss. For example, the propagation directions of 780-nm coupling and probe (or 795nm driving and signal) beams have an angle separation of about $0.9^{\circ}$, resulting in $L \Delta k=0.23$ radians in our system, where $L$ is the medium length, and a loss of $6 \%$. Fortunately, our study showed that a suitable two-photon detuning $(\delta)$ can compensate the phase mismatch. The experimental and theoretical studies of using $\delta$ to compensate a phase mismatch will be published elsewhere. Finally and most importantly, the efficiency of FWM process is sensitive to the groundstate decoherence rate $(\gamma)$ [29]. Thus, $\gamma$ must be as low as possible. The decoherence can be caused by stray magnetic fields in the transverse direction, frequency fluctuations of the two-photon Raman transition, inhomogeneity of the AC Stark shift, inhomogeneity of the longitudinal magnetic field, etc. Our works on the reduction of $\gamma$ can be found in Ref. [50]. In 
our system, $\gamma$ was mainly due to an unwanted but unavoidable transition of $\left|5 S_{1 / 2}, F=2\right\rangle \rightarrow\left|5 P_{3 / 2}, F^{\prime}=3\right\rangle$ driven by the coupling field with a large detuning of $266 \mathrm{MHz}$ or about $44 \Gamma$ $[49,56]$, where $\Gamma$ is the spontaneous decay rate of the excited state.

\section{APPENDIX B: THEORETICAL CALCULATION}

To characterize our experimental system and verify measurement outcomes, we made theoretical predictions with the OBEs of atomic density matrix and the MSEs of light fields given below [31,32].

$$
\begin{gathered}
\frac{\partial}{\partial t} \rho_{21}=\frac{i}{2} \Omega_{c}^{*} \rho_{31}+\frac{i}{2} \Omega_{d}^{*} \rho_{41}+i \delta \rho_{21}-\gamma \rho_{21}, \\
\frac{\partial}{\partial t} \rho_{31}=\frac{i}{2} \Omega_{p}+\frac{i}{2} \Omega_{c} \rho_{21}-\frac{\Gamma}{2} \rho_{31}, \\
\frac{\partial}{\partial t} \rho_{41}=\frac{i}{2} \Omega_{s}+\frac{i}{2} \Omega_{d} \rho_{21}+i \Delta \rho_{41}-\frac{\Gamma}{2} \rho_{41}, \\
\frac{1}{c} \frac{\partial}{\partial t} \Omega_{p}+\frac{\partial}{\partial z} \Omega_{p}=i \frac{\alpha}{2 L} \Gamma \rho_{31}, \\
\frac{1}{c} \frac{\partial}{\partial t} \Omega_{s}+\frac{\partial}{\partial z} \Omega_{s}=i \frac{\alpha}{2 L} \Gamma \rho_{41},
\end{gathered}
$$

where $\rho_{i j}$ is the element of density matrix operator of states $|i\rangle$ and $|j\rangle, \Omega_{c}, \Omega_{d}, \Omega_{p}$, and $\Omega_{s}$ are the Rabi frequencies of the coupling, driving, probe, and signal fields, $\delta$ is the two-photon detuning of the Raman transitions between two ground states $|1\rangle$ and $|2\rangle, \Delta$ is the one-photon detuning of the transition $|1\rangle \rightarrow|4\rangle$ or $|2\rangle \rightarrow|4\rangle, \gamma$ is the ground-state decoherence rate, $\Gamma$ is the spontaneous decay rate of the excited states $|3\rangle$ and $|4\rangle$ which is about $2 \pi \times 6 \mathrm{MHz}$ in the experiment, and $\alpha$ and $L$ are the OD and length of the medium. To achieve the above equations, we consider that the probe and signal fields are weak and can be treated as the perturbations of the system. The excited states $|3\rangle$ and $|4\rangle$ belong to the $D 2$ and $D 1$ lines of $\mathrm{Rb}$ atoms. The spontaneous decay rates of $|3\rangle$ and $|4\rangle$ as well as the transition strengths of $|1\rangle \rightarrow|3\rangle$ and $|1\rangle \rightarrow|4\rangle$ differ by about $5.3 \%$. In the theoretical calculation, we set the same decay rate for $\rho_{31}$ and $\rho_{41}$, and used the same OD for the probe and signal transitions. Since we always tuned the probe and coupling frequencies to the resonance frequencies of their transitions in the experiment, no one-photon detuning is present in Eq. (B2).

\section{APPENDIX C: DETERMINATION OF EXPERIMENTAL PARAMETERS}

We employed the measurement of slow light to determine the coupling Rabi frequency $\Omega_{c}$. A typically data of slow light of the probe pulse under the presence of the coupling field is shown in Fig. 5(a). According to the theory or theoretical predictions calculated with the OBEs and MSEs in Eqs. (B1), (B2), and (B4), the delay time of slow light is equal to $\alpha \Gamma / \Omega_{c}^{2}$ $[33,48]$. Once the $\mathrm{OD}(\alpha)$ is known which will be explained in the next paragraph, we can determine $\Omega_{c}$ from the delay time between input and output probe pulses. Attenuation of the probe pulse can be utilized to determine the decoherence rate $\gamma$, which was about $3 \times 10^{-3} \Gamma$ or $2 \pi \times 18 \mathrm{kHz}$ in this work. Furthermore, the condition of the coupling and driving fields

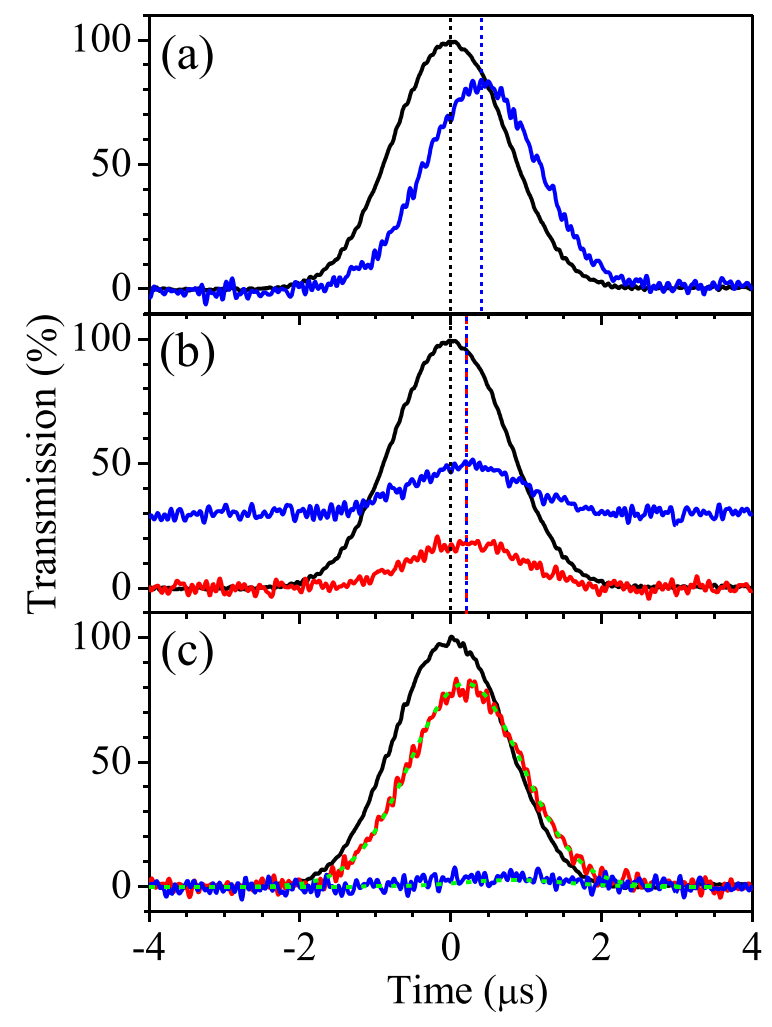

FIG. 5. Data of slow light in the single- $\Lambda$ and double- $\Lambda$ systems are shown in (a) and (b), respectively. Data of coherent wavelength conversion are presented in (c). In all of the data here, only the 780-nm probe pulse was sent to the input represented by black solid lines. Blue and red solid lines are the 780-nm probe and 795-nm signal pulses at the output. (a) Slow light of the probe pulse under the presence of only the coupling field. (b) Slow light of the probe and signal pulses under the presence of both of the coupling and driving fields at $\Delta=0$. Blue line is shifted up for clarity. The coupling Rabi frequencies in (a) and (b) were the same. Thus, the delay time in (b) became half of that in (a), indicating the coupling and driving fields had the same Rabi frequency, i.e., $\Omega_{c}=\Omega_{d}$. (c) At $\Delta /(2 \pi)=$ $-135 \mathrm{MHz}$ and $\Omega_{c}=\Omega_{d}$, the FWM process converted the input probe pulse nearly all to the output signal pulse. The 795-nm output transmission (ratio of output to input energies or photon numbers) was about $83 \%$, and the 780-nm one was less than $4 \%$. In (c), green dashed lines are the theoretical predictions calculated with $\alpha$ (OD) $=130, \Omega_{c}=\Omega_{d}=3.0 \Gamma$, and $\gamma$ (the ground-state decoherence rate) $=3 \times 10^{-3} \Gamma$. The values of $\alpha, \Omega_{c}\left(\Omega_{d}\right)$, and $\gamma$ were experimentally determined by the optimum one-photon detuning $\Delta_{\text {opt }}$ that maximizes the output signal pulse, the delay times in (a) and (b), and the ratio of output to input pulse amplitudes in (a), respectively.

having the same Rabi frequencies, i.e., $\Omega_{c}=\Omega_{d}$, was needed thorough all measurements of this work. We not only ensured $\Omega_{c}=\Omega_{d}$ by monitoring the powers of the coupling and driving fields, but also verified $\Omega_{c}=\Omega_{d}$ with the measurement of resonant FWM process (i.e., $\Delta=0$ ) as shown in Fig. 5(b). In the resonant FWM process, only the probe pulse was sent to the input, and both of the coupling and driving fields were present. From the theory or theoretical predictions calculated with the OBEs and MSEs in Eqs. (B1)-(B5), the delay time in the resonant FWM process is equal to $\alpha \Gamma /\left(\Omega_{c}^{2}+\Omega_{d}^{2}\right)[3,32]$. As the delay time in Fig. 5(b) became half of that in Fig. 5(a), 
we can know that $\Omega_{d}=\Omega_{c}$ and two fields have the same Rabi frequencies.

To determine the OD $(\alpha)$ of the experimental system, we employed the measurement of far-detuned FWM process, i.e., $|\Delta| \gg \Gamma$. In the far-detuned FWM process, only the probe pulse was sent to the input, and we measured the signal pulse at the output. In the measurement, we set $\Omega_{c}=\Omega_{d}$. The driving field had the one-photon detuning $\Delta$. According to the steady-state solution of Eqs. (B1)-(B5), the ratio of output signal (probe) field to input probe field under $\gamma=0$ is given by [31]

$$
\begin{aligned}
& \frac{\left|\Omega_{s}(z=L)\right|^{2}}{\left|\Omega_{p}(z=0)\right|^{2}}=\frac{1}{4}\left(1+e^{-2 A}-2 e^{-A} \cos \theta\right), \\
& \frac{\left|\Omega_{p}(z=L)\right|^{2}}{\left|\Omega_{p}(z=0)\right|^{2}}=\frac{1}{4}\left(1+e^{-2 A}+2 e^{-A} \cos \theta\right),
\end{aligned}
$$

where

$$
\begin{gathered}
\theta=\frac{\alpha}{2} \frac{\Delta / \Gamma}{1+(\Delta / \Gamma)^{2}}, \\
A=\frac{\alpha}{2} \frac{1}{1+(\Delta / \Gamma)^{2}}=\frac{\alpha-\sqrt{\alpha^{2}-16 \theta^{2}}}{4} .
\end{gathered}
$$

We now derive the criterion, which maximizes $\left|\Omega_{s}(L)\right|^{2}$ or equivalently minimizes $\left|\Omega_{p}(L)\right|^{2}$. Since Eq. (C1) or (C2) is a function of only $\alpha$ and $\Delta$, the OD of the system must be related to the optimum one-photon detuning $\Delta_{\text {opt }}$ at the maximum. Given $\Delta_{\text {opt }}$, we first take the derivative of Eq. (C1) with respect to $\alpha$, and obtained the criterion given by

$$
-e^{-A} \frac{d A}{d \alpha}+\cos \theta \frac{d A}{d \alpha}+\sin \theta \frac{d \theta}{d \alpha}=0 .
$$

The value of $\alpha$ in the experiment was large as compared with $\theta$, which is close to $\pi$ at $\Delta_{\text {opt }}$. Under $\alpha^{2} \gg 16 \theta^{2}, A \approx 2 \theta^{2} / \alpha$. Because $A$ is small and $\theta \approx \pi$, we then make the approximations of $e^{-A} \approx 1-A, \cos \theta \approx-1$, and $\sin \theta=\sin (\pi-\theta) \approx$ $\pi-\theta$. After $\theta$ and $A$ are substituted by the expressions in Eqs. (C3) and (C4), the criterion finally becomes

$$
\alpha \approx 2 \pi\left|\Delta_{\text {opt }} / \Gamma\right|-4 .
$$

Using the above equation and Eq. (C3), we also get $\theta \approx \pi-$ $2\left|\Gamma / \Delta_{\text {opt }}\right|$. On the other hand, the numerical calculations using Eqs. (B1)-(B5) with all possible experimental parameters in this work also conclude a formula of $\alpha \approx 6.2\left|\Delta_{\text {opt }} / \Gamma\right|-6$ with an uncertainty of $\pm 4 \%$. The value of $\alpha$ given by Eq. (C6) is consistent with that given by the numerical calculation. Therefore, by experimentally searching for $\Delta_{\text {opt }}$ we can determine the OD of the system. As an example, Fig. 5(c) shows the output signal pulse was maximized at $\Delta_{\text {opt }} /(2 \pi)=$ $-135 \pm 5 \mathrm{MHz}$. The measured $\Delta_{\text {opt }}$ indicates that the value of OD was $130 \pm 10$ in Fig. 5(c).

\section{APPENDIX D: A 50/50 FBS OPERATING WITH CLASSICAL LIGHT PULSES}

We properly adjusted the one-photon detuning $(\Delta)$ to produce a 50/50 FBS. Figure 6(a) shows the representative data that the 780-nm input pulse is split into the 780- and 795-nm output pulses of the very similar amount. At the split ratio of $\sim 0.48-0.51$, the average of data of output-to-input ratios

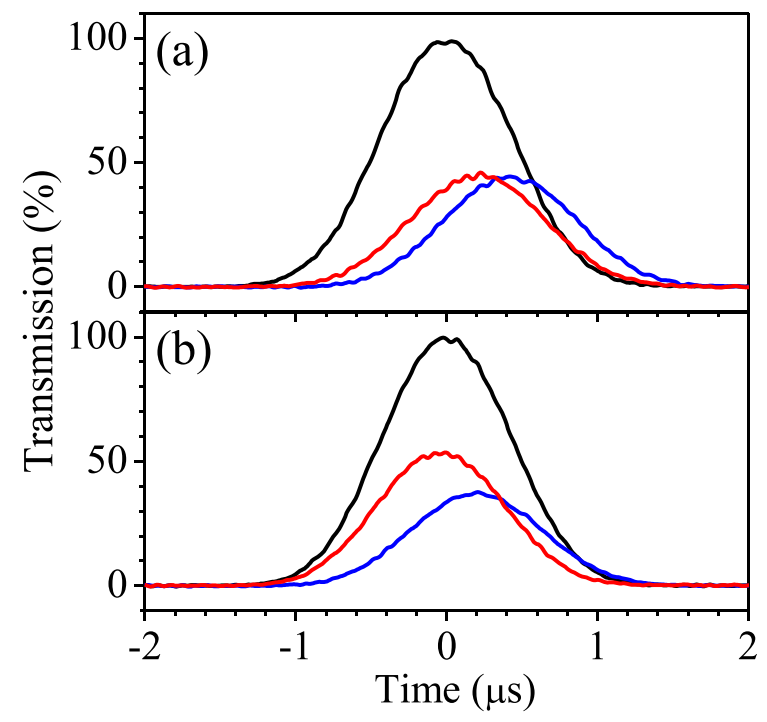

FIG. 6. A 50/50 frequency beam splitter operating with classical pulses. (a) At $\Delta /(2 \pi)=-205 \mathrm{MHz}, \alpha(\mathrm{OD})=110$, and $\Omega_{c}=\Omega_{d}$ $=3.0 \Gamma$, the FWM process converted the input $780-\mathrm{nm}$ probe (black) pulse to the output 795-nm signal (red) and 780-nm probe (blue) pulses with the transmissions of $46 \%$ and $45 \%$, respectively. The delay time between the red (or blue) and black lines is 0.21 (or 0.42 ) $\mu \mathrm{s}$. (b) Under the same experimental condition, the FWM process converted the input 795-nm (black) pulse to the output 795-nm (red) and 780-nm (blue) pulses with the transmissions of 53\% and 38\%, respectively. The delay time between the red (or blue) and black lines is -0.04 (or 0.20$) \mu \mathrm{s}$.

or total transmissions taken in different days gave $89 \pm 4 \%$, where the uncertainty is the standard deviation of the data. The values of OD in these measurements varied from 100 to 130 . Furthermore, we performed the measurement that only the 795-nm signal pulse was present at the input under the same experimental condition as Fig. 6(a). The total transmission of $93 \pm 2 \%$ with the split ratio of $\sim 0.37-0.46$ was obtained, where the split ratio here is the ratio of 780-nm output energy to total output energy. The representative data are shown in Fig. 6(b). With the same 50/50 FBS, the outcomes of the $780-\mathrm{nm}$ input differ from those of the 795-nm input, being caused by the nonnegligible decoherence rate in the system and the asymmetry between the $D 1$ and $D 2$ excited states. The output-to-input ratio or overall efficiency presented here, which accounts for the input coupling efficiency, attenuation due to propagation in the medium, and frequency conversion efficiency, is the best up-to-date record of FBS.

\section{APPENDIX E: BANDWIDTH OF THE FWM-BASED FBS}

The frequency conversion bandwidth of the FWM-based FBS is mainly determined by the EIT mechanism. The EIT bandwidth is approximately $\Omega_{c}^{2} /(\sqrt{\alpha} \Gamma)$. Under $\alpha=130$, $\Omega_{c}=\Omega_{d}=3.0 \Gamma$, and $\gamma=0.003 \Gamma$ in this work, the frequency converter has the maximum conversion efficiency of $84 \%$. This efficiency will drop to $42 \%$ with an input pulse of the bandwidth of $8 \mathrm{MHz}$, which is not much as compared with the bandwidth greater than $10 \mathrm{GHz}$ of the frequency conversions with fiber-based Bragg scattering [24] or PPLN 
waveguides [25]. Nevertheless, using a larger value of $\Omega_{c}$ helps the EIT bandwidth. In Ref. [44], the EIT bandwidth can be increased to $1.8 \mathrm{GHz}$ with a large coupling intensity. Considering that the coupling and driving Rabi frequencies (or powers) increase 10 (or 100) folds, i.e., $\Omega_{c}=\Omega_{d}=30 \Gamma$, and the values of $\alpha$ and $\gamma$ are kept the same. The predictions from the numerical calculation using Eqs. (B1)-(B5) show that the frequency conversion efficiency can be maximized to $87 \%$, and drop to $43.5 \%$ with an input pulse of the bandwidth of $450 \mathrm{MHz}$.

In this work, coherent-state single-photon pulses used in the measurement of frequency converter had the $e^{-1}$ full width of $2.4 \mu \mathrm{s}$, corresponding to the frequency bandwidth of $0.50 \mathrm{MHz}$. Recently, there are several schemes for realizing sources of narrow-linewidth single photons. For examples, single photons with linewidths of 4.5 and $6 \mathrm{MHz}$ were produced with solid-state systems $[59,60]$; those with linewidths of 0.38 and $1.9 \mathrm{MHz}$ were produced with atomic systems [57,58]. The issue of the bandwidth can be resolved by either increasing the powers of the coupling and driving fields, employing narrow-linewidth single photons, or both. Furthermore, the EIT mechanism is universal and can be applied to all kinds of media with different energy levels. As long as energy levels are available, the wavelength used in the EIT-based scheme can be flexible.

\section{APPENDIX F: FIDELITY OF FREQUENCY BEAM SPLITTER}

IN analogy to an ordinary BS, 780-nm (or 795-nm) input photons are reflected into 795-nm (or 780-nm) output photons and transmitted into 780-nm (795-nm) output photons by the FBS, with the split ratio defined by the ratio of reflected output photon number to total output photon number. The fidelity $F$ is the important issue and can be determined by the following formula $[6,51,52]$ :

$$
F=\frac{\left|\operatorname{Tr}\left[\hat{V}^{\dagger} \hat{U}\right]\right|^{2}}{4 T},
$$

where $\hat{U}$ represents the operator of the ideal BS, $\hat{V}$ represents that of the FBS in this study, $\operatorname{Tr}[\ldots]$ means the operation of trace, and $T$ is the total transmission of $\hat{V}$. One can write down the general expression of a FBS as

$$
\hat{V}=\left[\begin{array}{cc}
t_{1} & r_{2} e^{i \phi_{2}} \\
r_{1} e^{i \phi_{1}} & t_{2}
\end{array}\right],
$$

where $t_{1}$ (or $t_{2}$ ) and $r_{1}$ (or $r_{2}$ ) are the transmission and reflection coefficients of input 1 (or input 2) and $\phi_{1}$ (or $\phi_{2}$ ) is the phase difference between the reflected and transmitted outputs. Because of loss, $t_{1}^{2}+r_{1}^{2} \leqslant 1$ and $t_{2}^{2}+r_{2}^{2} \leqslant 1$. The split ratio of two inputs are determined by $r_{1}^{2} /\left(t_{1}^{2}+r_{1}^{2}\right)$ and $r_{2}^{2} /\left(t_{2}^{2}+r_{2}^{2}\right)$ and can be in general different.

\section{Frequency beam splitter with tunable split ratio}

We consider the case that the ground-state decoherence rate in the system is negligible, i.e., $\gamma=0$. The steady-state solution of Eqs. (B1)-(B5) under $\Omega_{c}=\Omega_{d}$ provides $t_{1}, r_{1}, \phi_{1}$, $t_{2}, r_{2}$ and $\phi_{2}$ for $\hat{V}$ of the FWM-based FBS, which is given by

$$
\hat{V}=\frac{1}{2}\left[\begin{array}{ll}
V_{11} & V_{12} \\
V_{21} & V_{22}
\end{array}\right],
$$

where

$$
\begin{gathered}
V_{11}=V_{22}=\sqrt{1+e^{-2 A}+2 e^{-A} \cos \theta}, \\
V_{12}=V_{21}=e^{i \phi / 2} \sqrt{1+e^{-2 A}-2 e^{-A} \cos \theta}, \\
A=\frac{\alpha-\sqrt{\alpha^{2}-16 \theta^{2}}}{4}, \\
\phi=2 \tan ^{-1}\left(\frac{2 e^{-A}}{1-e^{-2 A}} \sin \theta\right) .
\end{gathered}
$$

Equation (C3) shows $\theta$ is a function of $\mathrm{OD}(\alpha)$ and one-photon detuning $(\Delta)$. According to $t_{1}$ and $r_{1}$ (or $t_{2}$ and $r_{2}$ ) in Eq. (F3), the split ratio is related to $\theta$ as

$$
s=\frac{1}{2}\left(1-\frac{2 e^{-A}}{1+e^{-2 A}} \cos \theta\right) .
$$

One can immediately see that $\theta \approx \pi$ makes $s \rightarrow 1$ under a small $A$ (i.e., a large $\alpha$ ), and $\theta=\pi / 2$ exactly makes $s=0.5$. Based on Eq. (F3), the total transmission of FBS is

$$
T=\frac{1+e^{-2 A}}{2} \text {. }
$$

The ideal BS must have $\phi_{1}+\phi_{2}=\pi$ in Eq. (F2) [53,54]. Corresponding to $\hat{V}$ in Eq. (F3) of $\phi_{1}=\phi_{2}=\phi$ and the split ratio of $s$, the operator $\hat{U}$ of ideal BS is given by

$$
\hat{U}=\left[\begin{array}{cc}
\sqrt{1-s} & i \sqrt{s} \\
i \sqrt{s} & \sqrt{1-s}
\end{array}\right] .
$$

With $\hat{V}$ in Eq. (F3) and the above $\hat{U}$, Eq. (F1) becomes

$$
\begin{aligned}
F= & \frac{1}{4 T}\left[\left(1+e^{-2 A}\right)+2(1-2 s) e^{-A} \cos \theta\right. \\
& \left.+2 \sqrt{s(1-s)} \sqrt{\left(1+e^{-2 A}\right)^{2}-4 e^{-2 A} \cos ^{2} \theta} \sin \left(\frac{\phi}{2}\right)\right] .
\end{aligned}
$$

\section{2. $50 / 50$ frequency beam splitter}

We again consider the case that the ground-state decoherence rate in the system is negligible, i.e., $\gamma=0$. The 50/50 FBS has $\theta=\pi / 2$, making $\cos \theta=0$ and $s=0.5$, as indicated by Eq. (F8). Using $\cos \theta=0$ in Eq. (F10), we find that the fidelity of 50/50 FBS is

$$
F=\frac{1}{2}+\frac{1}{2} \sin \left(\frac{\phi}{2}\right)
$$

Since the value of $\alpha$ (OD) in our experiment was large as compared with $\theta, A \approx 2 \theta^{2} / \alpha=\pi^{2} /(2 \alpha) \ll 1$ in Eq. (F6). We set $\theta=\pi / 2$ in Eq. (F7) to get $\sin (\phi / 2)$, and utilize $A \ll 1$ to obtain

$$
F=\frac{1}{2}+\frac{e^{-A}}{1+e^{-2 A}} \approx 1-\frac{A^{2}}{4} \approx 1-\frac{\pi^{4}}{16 \alpha^{2}} .
$$


Using $A \ll 1$ in Eq. (F9),

$$
T \approx 1-A=1-\frac{\pi^{2}}{2 \alpha} .
$$

Therefore, a larger OD not only results in a lower loss, but also makes the fidelity better.

\section{Realistic frequency beam splitter}

In reality, the ground-state decoherence rate $\gamma$ in the experimental system was not negligible. A nonzero $\gamma$ makes two inputs of the FWM-based FBS produce different split ratios, i.e., $r_{1}^{2} /\left(t_{1}^{2}+r_{1}^{2}\right) \neq r_{2}^{2} /\left(t_{2}^{2}+r_{2}^{2}\right)$, and different phases, i.e., $\phi_{1} \neq \phi_{2}$. Corresponding to the general expression of $\hat{V}$ in Eq. (F2) of nonzero $\Delta \phi=\phi_{1}-\phi_{2}$, the operator $\hat{U}$ of ideal $50 / 50 \mathrm{BS}$ is given by

$$
\hat{U}=\frac{1}{\sqrt{2}}\left[\begin{array}{cc}
1 & e^{i(\pi-\Delta \phi) / 2} \\
e^{i(\pi+\Delta \phi) / 2} & 1
\end{array}\right] .
$$

The above expressions of phases in the off-diagonal terms ensure that the ideal BS has $\phi_{1}+\phi_{2}=\pi$. With $\hat{V}$ in Eq. (F2) and the above $\hat{U}$, the fidelity in Eq. (F1) becomes

$$
F=\frac{1}{2}+\frac{\bar{t} \bar{r}}{T} \sin \left(\frac{\phi}{2}\right),
$$

where $\bar{t}=\left(t_{1}+t_{2}\right) / 2, \bar{r}=\left(r_{1}+r_{2}\right) / 2, T=\bar{t}^{2}+\bar{r}^{2}$, and $\phi=$ $\phi_{1}+\phi_{2}$.

\section{APPENDIX G: DETERMINATION OF THE PHASE OF FREQUENCY BEAM SPLITTER WITH HOMI}

The phase $\phi$ in Eq. (F10) is the sum of the phase difference between the reflected and transmitted waves of input $1, \phi_{1}$, and that of input $2, \phi_{2}$, in Eq. (F2), i.e., $\phi=\phi_{1}+\phi_{2}$. The following example illustrates how the measurement of $g^{(2)}$ in the HOMI can determine $\phi$. A BS is represented by the matrix in Eq. (F2). In the HOMI, two single photons of the Fock state are sent to the two input ports of BS. The value of $g^{(2)}$ of the two output ports of BS is defined by

$$
g^{(2)} \equiv \frac{\left\langle N_{1} N_{2}\right\rangle}{\left\langle N_{1}\right\rangle\left\langle N_{2}\right\rangle},
$$

where $\left\langle N_{1}\right\rangle$ or $\left\langle N_{2}\right\rangle$ is the photon count of each output mode, and $\left\langle N_{1} N_{2}\right\rangle$ is the photon-photon coincidence count between the two output modes. Because of the energy (photon number) conservation, $\left\langle N_{1}\right\rangle=t_{1}^{2}+r_{2}^{2}$ and $\left\langle N_{2}\right\rangle=t_{2}^{2}+r_{1}^{2}$, where $t_{1}\left(t_{2}\right)$ and $r_{1}\left(r_{2}\right)$ are the transmission and reflection coefficients of input 1 (input 2). The major outcomes of the BS correspond to the following two-mode wave functions of $t_{1} t_{2}|1,1\rangle$, $t_{1} r_{2} e^{i \phi_{2}} \sqrt{2}|2,0\rangle, t_{2} r_{1} e^{i \phi_{1}} \sqrt{2}|0,2\rangle$, and $r_{1} r_{2} e^{i \phi}|1,1\rangle$, where the first and second quanta represent photon numbers in two output modes. Only the wave functions of $|1,1\rangle$ can contribute to $\left\langle N_{1} N_{2}\right\rangle$, which is equal to $\left(t_{1} t_{2}+r_{1} r_{2} e^{-i \phi}\right)\left(t_{1} t_{2}+r_{1} r_{2} e^{i \phi}\right)$, and those of $|2,0\rangle$ and $|0,2\rangle$ do not make any contribution. Other possible output wave functions are proportional to $|1,0\rangle$, $|0,1\rangle$, and $|0,0\rangle$, which do not contribute to $\left\langle N_{1} N_{2}\right\rangle$, either. Once knowing $\left\langle N_{1}\right\rangle,\left\langle N_{2}\right\rangle$, and $\left\langle N_{1} N_{2}\right\rangle$ [54], we obtain

$$
g^{(2)}=\frac{t_{1}^{2} t_{2}^{2}+r_{1}^{2} r_{2}^{2}+2 t_{1} t_{2} r_{1} r_{2} \cos \phi}{\left(t_{1}^{2}+r_{2}^{2}\right)\left(t_{2}^{2}+r_{1}^{2}\right)} .
$$

Therefore, given the BS's $t_{1}, r_{1}, t_{2}$, and $r_{2}$, one can determine $\phi$ from the value of $g^{(2)}$ as demonstrated by the above equation.

A $50 / 50$ BS has $t_{1}=r_{1}=t_{2}=r_{2}$. Based on Eq. (G2), $g^{(2)}=(1+\cos \phi) / 2$ revealing that the two-photon event corresponding to $t_{1} t_{2}|1,1\rangle$ (both input photons transmit through the BS) interferes with that corresponding to $r_{1} r_{2} e^{i \phi}|1,1\rangle$ (both are reflected by the BS). An ideal 50/50 BS further has $\phi=\pi[53,54]$, making $g^{(2)}=0$ which is exactly the consequence that the interference is completely destructive. With two Fock-state single photons at two input ports of the ideal BS in the HOMI, it is well known that both photons always emerge together at one of the output ports (corresponding to $|2,0\rangle$ or $|0,2\rangle)$ as depicted in Fig. 1(c), resulting in $g^{(2)}=0$ $[38,39]$.

\section{APPENDIX H: HOMI MEASUREMENT WITH COHERENT-STATE PHOTONS}

In this section, we will derive the value of $g^{(2)}$ in the HOMI of a realistic BS with coherent-state light. To make the theoretical situation consistent with the experimental condition of our HOMI measurement, we consider that the phases of the two input coherent states are completely uncorrelated, and the mean photon numbers of the two states are the same. Equation (F2) represents the operator of realistic BS. Two coherent states $|\beta\rangle$ and $\left|\beta e^{i \xi}\right\rangle$ are incident to inputs 1 and 2 of the BS, respectively, where $|\beta|^{2}$ indicates the mean photon number in either state and $\xi$ is the relative phase between the two states. Since the two coherent states are phase-uncorrelated, we will average $\xi$ over all phases later.

In Eq. (F2), the transmission and reflection coefficients of input 1 (or input 2) of the BS are $t_{1}$ (or $t_{2}$ ) and $r_{1}$ (or $r_{2}$ ), respectively, and the phase difference between the reflected and transmitted light is $\phi_{1}$ (or $\phi_{2}$ ). Thus, the two wave functions emerging at output 1 and 2 of the BS are given by

$$
\begin{aligned}
& \psi_{1}=\left|t_{1} \beta+\left(r_{2} e^{i \phi_{2}}\right) \beta e^{i \xi}\right\rangle_{1}=\left|\left[t_{1}+r_{2} e^{i\left(\phi_{2}+\xi\right)}\right] \beta\right\rangle_{1}, \\
& \psi_{2}=\left|\left(r_{1} e^{i \phi_{1}}\right) \beta+t_{2} \beta e^{i \xi}\right\rangle_{2}=\left|\left(r_{1} e^{i \phi_{1}}+t_{2} e^{i \xi}\right) \beta\right\rangle_{2},
\end{aligned}
$$

where the subscripts 1 and 2 indicate the output modes 1 and 2. Let us denote $\left\langle N_{1}\right\rangle$ and $\left\langle N_{2}\right\rangle$ as the photon counts of outputs 1 and 2 of the BS, and $\left\langle N_{1} N_{2}\right\rangle$ as the photon-photon coincidence count between the two outputs. The value of $g^{(2)}$ is defined by

$$
g^{(2)} \equiv \frac{\left\langle N_{1} N_{2}\right\rangle}{\left\langle N_{1}\right\rangle\left\langle N_{2}\right\rangle} .
$$

With $\psi_{1}$ and $\psi_{2}$, we can evaluate $\left\langle N_{1}\right\rangle$ and $\left\langle N_{2}\right\rangle$ with the photon number operators $a_{1}^{\dagger} a_{1}$ and $a_{2}^{\dagger} a_{2}$ in the following:

$$
\begin{aligned}
& \left\langle N_{1}\right\rangle=\frac{1}{2 \pi} \int_{0}^{2 \pi} d \xi \psi_{1}^{\dagger}\left(a_{1}^{\dagger} a_{1}\right) \psi_{1}=\left(t_{1}^{2}+r_{2}^{2}\right)|\beta|^{2}, \\
& \left\langle N_{2}\right\rangle=\frac{1}{2 \pi} \int_{0}^{2 \pi} d \xi \psi_{2}^{\dagger}\left(a_{2}^{\dagger} a_{2}\right) \psi_{2}=\left(r_{1}^{2}+t_{2}^{2}\right)|\beta|^{2} .
\end{aligned}
$$


The two-mode wave function at the output, $\psi_{12}$, is the direct product of $\psi_{1}$ and $\psi_{2}$, i.e., $\psi_{12}=\psi_{1} \otimes \psi_{2}$. Hence, the value of $\left\langle N_{1} N_{2}\right\rangle$ is given by

$$
\begin{aligned}
\left\langle N_{1} N_{2}\right\rangle= & \frac{1}{2 \pi} \int_{0}^{2 \pi} d \xi \psi_{12}^{\dagger}\left(a_{1}^{\dagger} a_{1} a_{2}^{\dagger} a_{2}\right) \psi_{12} \\
= & {\left[\left(t_{1}^{2}+r_{2}^{2}\right)\left(r_{1}^{2}+t_{2}^{2}\right)\right.} \\
& \left.+2 t_{1} t_{2} r_{1} r_{2} \cos \left(\phi_{1}+\phi_{2}\right)\right]|\beta|^{4} .
\end{aligned}
$$

Knowing the values of $\left\langle N_{1}\right\rangle,\left\langle N_{2}\right\rangle$, and $\left\langle N_{1} N_{2}\right\rangle$, we obtain

$$
g^{(2)}=1+\frac{2 t_{1} t_{2} r_{1} r_{2}}{\left(t_{1}^{2}+r_{2}^{2}\right)\left(t_{2}^{2}+r_{1}^{2}\right)} \cos \phi,
$$

where $\phi=\phi_{1}+\phi_{2}$. Both $g^{(2)}$ and fidelity, $F$, are related to $\phi$ as demonstrated by the above equation and Eq. (F15). Therefore, given $t_{1}, r_{1}, t_{2}$, and $r_{2}$ of a realistic BS, one can determine the fidelity of this BS from the value of $g^{(2)}$ in the HOMI measurement. With two coherent-state single photons, one can also show that the HOMI of ideal 50/50 BS results in $g^{(2)}=0.5[40-42]$.

As the example shown in Fig. 3(b) of the main text, the probability waveform of probe (signal) photons in the experiment was the Gaussian pulse, and the background noise was just a constant baseline or offset. The counts of background noise were nearly all contributed from the leakages of strong coupling and driving fields. With and without the presence of the atoms, these background counts were almost the same. Besides, there was no observable difference between the background counts with and without the presence of single-photon or few-photon probe (signal) pulse.

The background noise can degrade the measured value of photon-photon correlation function, $g_{m}^{(2)}$, given by

$$
g_{m}^{(2)}=\frac{\left\langle N_{1}^{\prime} N_{2}^{\prime}\right\rangle}{\left\langle N_{1}^{\prime}\right\rangle\left\langle N_{2}^{\prime}\right\rangle},
$$

where

$$
\begin{gathered}
\left\langle N_{1}^{\prime}\right\rangle=\left\langle N_{1}\right\rangle+n_{1}, \\
\left\langle N_{2}^{\prime}\right\rangle=\left\langle N_{2}\right\rangle+n_{2}, \\
\left\langle N_{1}^{\prime} N_{2}^{\prime}\right\rangle=\left\langle N_{1} N_{2}\right\rangle+n_{1}\left\langle N_{2}\right\rangle+n_{2}\left\langle N_{1}\right\rangle+n_{1} n_{2} .
\end{gathered}
$$

In the above equations, $n_{1}$ and $n_{2}$ denote the counts of probe and signal SPCMs contributed from the background noises, which are uncorrelated to each other and also uncorrelated to the probe and signal counts. Since $n_{1}$ and $n_{2}$ are small as compared with $\left\langle N_{1}\right\rangle$ and $\left\langle N_{2}\right\rangle$, we consider $g_{m}^{(2)}$ up to the first orders of $n_{1}$ and $n_{2}$, and obtain

$$
g_{m}^{(2)} \approx g^{(2)}+\left[1-g^{(2)}\right]\left(\frac{n_{1}}{\left\langle N_{1}\right\rangle}+\frac{n_{2}}{\left\langle N_{2}\right\rangle}\right) .
$$

The background noise is a constant offest of the Gaussian pulse, which can be clearly identified from the data. Hence, one can directly determine the ratios of $n_{1} /\left\langle N_{1}\right\rangle$ and $n_{2} /\left\langle N_{2}\right\rangle$ based on the data, and correct the measured $g_{m}^{(2)}$ to get the actual or corrected $g^{(2)}$. The values of photon-photon correlation function shown in Fig. 4 of the main text were all corrected for the background noise, where $n_{1} /\left\langle N_{1}\right\rangle$ of the $780-\mathrm{nm}$ probe SPCM and $n_{2} /\left\langle N_{2}\right\rangle$ of the 795-nm signal SPCM were about $5 \%$ and $13 \%$, respectively.
[1] A. Eckstein, B. Brecht, and C. Silberhorn, A quantum pulse gate based on spectrally engineered sum frequency generation, Opt. Express 19, 13770 (2011).

[2] B. Brecht, A. Eckstein, R. Ricken, V. Quiring, H. Suche, L. Sansoni, and C. Silberhorn, Demonstration of coherent timefrequency Schmidt mode selection using dispersion-engineered frequency conversion, Phys. Rev. A 90, 030302(R) (2014)

[3] M.-J. Lee, J. Ruseckas, C.-Y. Lee, V. Kudrisov, K.-F. Chang, H.-W. Cho, G. Juzeliūnas, and I. A. Yu, Experimental demonstration of spinor slow light, Nat. Commun. 5, 5542 (2014).

[4] J. M. Lukens and P. Lougovski, Frequency-encoded photonic qubits for scalable quantum information processing, Optica 4, 8 (2017).

[5] T. Kobayashi, R. Ikuta, S. Yasui, S. Miki, T. Yamashita, H. Terai, T. Yamamoto, M. Koashi, and N. Imoto, Frequencydomain Hong-Ou-Mandel interference, Nat. Photon. 10, 441 (2016).

[6] H.-H. Lu, J. M. Lukens, N. A. Peters, O. D. Odele, D. E. Leaird, A. M. Weiner, and P. Lougovski, Electro-Optic Frequency Beam Splitters and Tritters for High-Fidelity Photonic Quantum Information Processing, Phys. Rev. Lett. 120, 030502 (2018).
[7] J. Brendel, N. Gisin, W. Tittel, and H. Zbinden, Pulsed Energy-Time Entangled Twin-Photon Source for Quantum Communication, Phys. Rev. Lett. 82, 2594 (1999).

[8] N. T. Islam, C. C. W. Lim, C. Cahall, J. Kim, and D. J. Gauthier, Provably secure and high-rate quantum key distribution with time-bin qudits, Sci. Adv. 3, e1701491 (2017).

[9] J. P. Lee, L. M. Wells, B. Villa, S. Kalliakos, R. M. Stevenson, D. J. P. Ellis, I. Farrer, D. A. Ritchie, A. J. Bennett, and A. J. Shields, Controllable Photonic Time-Bin Qubits from a Quantum Dot, Phys. Rev. X 8, 021078 (2018).

[10] H.-P. Lo, T. Ikuta, N. Matsuda, T. Honjo, and H. Takesue, Entanglement generation using a controlled-phase gate for time-bin qubits, Appl. Phys. Expr. 11, 092801 (2018).

[11] A. Tchebotareva, S. L. N. Hermans, P. C. Humphreys, D. Voigt, P. J. Harmsma, L. K. Cheng, A. L. Verlaan, N. Dijkhuizen, W. de Jong, A. Dréau, and R. Hanson, Entanglement between a Diamond Spin Qubit and a Photonic Time-Bin Qubit at Telecom Wavelength, Phys. Rev. Lett. 123, 063601 (2019).

[12] J. Fiurášek, Interconversion between single-rail and dual-rail photonic qubits, Phys. Rev. A 95, 033802 (2017).

[13] P. Vernaz-Gris, K. Huang, M. Cao, A. S. Sheremet, and J. Laurat, Highly-efficient quantum memory for polarization 
qubits in a spatially-multiplexed cold atomic ensemble, Nat. Commun. 9, 363 (2018).

[14] D. Drahi, D. V. Sychev, K. K. Pirov, E. A. Sazhina, V. A. Novikov, I. A. Walmsley, and A. I. Lvovsky, Quantum interface between single- and dual-rail optical qubits, arXiv:1905.08562 [quant-ph].

[15] L. M. Duan, M. D. Lukin, J. I. Cirac, and P. Zoller, Longdistance quantum communication with atomic ensembles and linear optics, Nature 414, 413 (2001).

[16] N. K. Langford, S. Ramelow, R. Prevedel, W. J. Munro, G. J. Milburn, and A. Zeilinger, Efficient quantum computing using coherent photon conversion, Nature 478, 360 (2011).

[17] P. Kumar, Quantum frequency conversion, Opt. Lett. 15, 1476 (1990).

[18] S. Tanzilli, W. Tittel, M. Halder, O. Alibart, P. Baldi, N. Gisin, and $\mathrm{H}$. Zbinden, A photonic quantum information interface, Nature 437, 116 (2005).

[19] M. T. Rakher, L. Ma, O. Slattery, X. Tang, and K. Srinivasan, Quantum transduction of telecommunications-band single photons from a quantum dot by frequency upconversion, Nat. Photon. 4, 786 (2010).

[20] H. J. McGuinness, M. G. Raymer, C. J. McKinstrie, and S. Radic, Quantum Frequency Translation of Single-Photon States in a Photonic Crystal Fiber, Phys. Rev. Lett. 105, 093604 (2010).

[21] S. Ates, I. Agha, A. Gulinatti, I. Rech, M. T. Rakher, A. Badolato, and K. Srinivasan, Two-Photon Interference Using Background-Free Quantum Frequency Conversion of Single Photons Emitted by an InAs Quantum Dot, Phys. Rev. Lett. 109, 147405 (2012).

[22] A. S. Clark, S. Shahnia, M. J. Collins, C. Xiong, and B. J. Eggleton, High-efficiency frequency conversion in the singlephoton regime, Opt. Lett. 38, 947 (2013).

[23] Q. Li, M. Davanço, and K. Srinivasan, Efficient and low-noise single-photon-level frequency conversion interfaces using silicon nanophotonics, Nat. Photon. 10, 406 (2016).

[24] S. Clemmen, A. Farsi, S. Ramelow, and A. Gaeta, Ramsey Interference with Single Photons, Phys. Rev. Lett. 117, 223601 (2016); A. Farsi, Ph.D. thesis, Cornell University (2015).

[25] N. Maring, D. Lago-Rivera, A. Lenhard, G. Heinze, and H. de Riedmatten, Quantum frequency conversion of memorycompatible single photons from $606 \mathrm{~nm}$ to the telecom C-band, Optica 5, 507 (2018).

[26] A. G. Radnaev, Y. O. Dudin, R. Zhao, H. H. Jen, S. D. Jenkins, A. Kuzmich, and T. A. B. Kennedy, A quantum memory with telecom-wavelength conversion, Nat. Phys. 6, 894 (2010).

[27] G. Wang, Y. Xue, J.-H. Wu, Z.-H. Kang, Y. Jiang, S.-S. Liu, and J.-Y. Gao, Efficient frequency conversion induced by quantum constructive interference, Opt. Lett. 35, 3778 (2010).

[28] Z.-Y. Liu, J.-T. Xiao, J.-K. Lin, J.-J. Wu, J.-Y. Juo, C.-Y. Cheng, and Y.-F. Chen, High-efficiency backward four-wave mixing by quantum interference, Sci. Rep. 7, 15796 (2017).

[29] M. Jain, H. Xia, G. Y. Yin, J. Merriam, and S. E. Harris, Efficient Nonlinear Frequency Conversion with Maximal Atomic Coherence, Phys. Rev. Lett. 77, 4326 (1996).

[30] M. G. Payne and L. Deng, Consequences of induced transparency in a double- $\Lambda$ scheme: Destructive interference in four-wave mixing, Phys. Rev. A 65, 063806 (2002).
[31] C.-K. Chiu, Y.-H. Chen, Y.-C. Chen, I. A. Yu, Y.-C. Chen, and Y.-F. Chen, Low-light-level four-wave mixing by quantum interference, Phys. Rev. A 89, 023839 (2014).

[32] C.-Y. Lee, B.-H. Wu, G. Wang, Y.-F. Chen, Y.-C. Chen, and I. A $\mathrm{Yu}$, High conversion efficiency in resonant four-wave mixing processes, Opt. Express 24, 1008 (2016).

[33] M. Fleischhauer, A. Imamoglu, and J. Marangos, Electromagnetically induced transparency: Optics in coherent media, Rev. Mod. Phys. 77, 633 (2005).

[34] A. M. Childs, I. L. Chuang, and D. W. Leung, Realization of quantum process tomography in NMR, Phys. Rev. A 64, 012314 (2001).

[35] J. L. O’Brien, G. J. Pryde, A. Gilchrist, D. F. James, N. K. Langford, T. C. Ralph, and A. G. White, Quantum process tomography of a controlled-NOT gate, Phys. Rev. Lett. 93, 080502 (2004).

[36] R. C. Bialczak, M. Ansmann, M. Hofheinz, E. Lucero, M. Neeley, A. D. O'Connell, D. Sank, H. Wang, J. Wenner, M. Steffen, A. N. Cleland, and J. M. Martinis, Quantum process tomography of a universal entangling gate implemented with Josephson phase qubits, Nat. Phys. 6, 409 (2010).

[37] Y. Kim, Y.-S. Kim, S.-Y. Lee, S.-W. Han, S. Moon, Y.-H Kim, and Y.-W. Cho, Direct quantum process tomography via measuring sequential weak values of incompatible observables, Nat. Commun. 9, 192 (2018).

[38] C. K. Hong, Z. Y. Ou, and L. Mandel, Measurement of Subpicosecond Time Intervals between Two Photons by Interference, Phys. Rev. Lett. 59, 2044 (1987).

[39] T. B. Pittman, D. V. Strekalov, A. Migdall, M. H. Rubin, A. V. Sergienko, and Y. H. Shih, Can Two-Photon Interference be Considered the Interference of Two Photons? Phys. Rev. Lett. 77, 1917 (1996).

[40] J. G. Rarity, P. R. Tapster, and R. Loudon, Non-classical interference between independent sources, J. Opt. B: Quant. Semiclass. Opt. 7, S171 (2005).

[41] Y.-S. Kim, O. Slattery, P. S. Kuo, and X. Tang, Conditions for two-photon interference with coherent pulses, Phys. Rev. A 87, 063843 (2013).

[42] H. Chen, X.-B. An, J. Wu, Z.-Q. Yin, S. Wang, W. Chen, and Z.-F. Han, Hong-Ou-Mandel interference with two independent weak coherent states, Chin. Phys. B 25, 020305 (2016).

[43] S. Marcinkevičius, A. Gushterov, and J. P. Reithmaier, Transient electromagnetically induced transparency in selfassembled quantum dots, Appl. Phys. Lett. 92, 041113 (2008).

[44] E. Ignesti, R. Buffa, L. Fini, E. Sali, M. V. Tognetti, S. Cavalieri, Controlling the propagation of broadband light pulses by Electromagnetically Induced Transparency, Opt. Commun. 285 , 1185 (2012).

[45] V. M. Acosta, K. Jensen, C. Santori, D. Budker, and R. G. Beausoleil, Electromagnetically Induced Transparency in a Diamond Spin Ensemble Enables All-Optical Electromagnetic Field Sensing, Phys. Rev. Lett. 110, 213605 (2013).

[46] R. Akhmedzhanov, L. Gushchin, N. Nizov, V. Nizov, D. Sobgayda, I. Zelensky, and A. Kalachev, Electromagnetically induced transparency in an isotopically purified $\mathrm{Nd}^{3+}: \mathrm{YLiF}_{4}$ crystal, Phys. Rev. B 97, 245123 (2018).

[47] J. Long, H. S. Ku, X. Wu, X. Gu, R. E. Lake, M. Bal, Y.-X. Liu, and D. P. Pappas, Electromagnetically Induced Transparency in Circuit Quantum Electrodynamics with Nested Polariton States, Phys. Rev. Lett. 120, 083602 (2018). 
[48] Y.-W. Lin, H.-C. Chou, P. P. Dwivedi, Y.-C. Chen, and I. A. Yu, Using a pair of rectangular coils in the MOT for the production of cold atom clouds with large optical density, Opt. Express 16, 3753 (2008).

[49] Y.-H. Chen, M.-J. Lee, I.-C. Wang, S. Du, Y.-F. Chen, Y.-C. Chen, and I. A. Yu, Coherent Optical Memory with High Storage Efficiency and Large Fractional Delay, Phys. Rev. Lett. 110, 083601 (2013).

[50] Y.-H. Chen, M.-J. Lee, I.-C. Wang, and I. A. Yu, Fidelity of electromagnetically-induced-transparency-based optical memory, Phys. Rev. A 88, 023805 (2013).

[51] D. B. Uskov, L. Kaplan, A. M. Smith, S. D. Huver, and J. P. Dowling, Maximal success probabilities of linear-optical quantum gates, Phys. Rev. A 79, 042326 (2009).

[52] S. Rahimi-Keshari, M. A. Broome, R. Fickler, A. Fedrizzi, T. C. Ralph, and A. G. White, Direct characterization of linear-optical networks, Opt. Express 21, 13450 (2013).

[53] S. M. Barnett, J. Jeffers, and A. Gatti, Quantum optics of lossy beam splitters, Phys. Rev. A 57, 2134 (1998).

[54] R. Uppu, T. A. W. Wolterink, T. B. H. Tentrup, and P. W. H. Pinkse, Quantum optics of lossy asymmetric beam splitters, Opt. Express 24, 16440 (2016).

[55] N. Lauk, C. O'Brien, and M. Fleischhauer, Fidelity of photon propagation in electromagnetically induced transparency in the presence of four-wave mixing, Phys. Rev. A 88, 013823 (2013).
[56] Y.-F. Hsiao, P.-J. Tsai, H.-S. Chen, S.-X. Lin, C.-C. Hung, C.-H. Lee, Y.-H. Chen, Y.-F. Chen, I. A. Yu, and Y.-C. Chen, Highly Efficient Coherent Optical Memory Based on Electromagnetically Induced Transparency, Phys. Rev. Lett. 120, 183602 (2018).

[57] Z. Han, P. Qian, L. Zhou, J. F. Chen, and W. Zhang, Coherence time limit of the biphotons generated in a dense cold atom cloud, Sci. Rep. 5, 9126 (2015).

[58] L. Zhu, X. Guo, C. Shu, H. Jeong, and S. Du, Bright narrowband biphoton generation from a hot rubidium atomic vapor cell, Appl. Phys. Lett. 110, 161101 (2017).

[59] C.-H. Wu, T.-Y. Wu, Y.-C. Yeh, P.-H. Liu, C.-H. Chang, C.-K Liu, T. Cheng, and C.-S. Chuu, Bright single photons for lightmatter interaction, Phys. Rev. A 96, 023811 (2017).

[60] P.-J. Tsai and Y.-C. Chen, Ultrabright, narrow-band photon-pair source for atomic quantum memories, Quant. Sci. Technol. 3, 034005 (2018).

[61] S.-W. Su, Y.-H. Chen, S.-C. Gou, T.-L. Horng, and I. A. Yu, Dynamics of slow light and light storage in a Doppler-broadened electromagnetically-induced-transparency medium: A numerical approach, Phys. Rev. A 83, 013827 (2011).

[62] S.-W. Su, Y.-H. Chen, S.-C. Gou, and I. A. Yu, An effective thermal-parametrization theory for the slow-light dynamics in a Doppler-broadened electromagnetically induced transparency medium, J. Phys. B 44, 165504 (2011). 